\title{
MICROTEXTURE CHARACTERIZATION BY EBSP IN IRON AND TITANIUM ALLOYS
}

\author{
R. PENELLE and T. BAUDIN \\ Laboratoire de Métallurgie Structurale, Bât 413, URA CNRS 1107, Université \\ Paris-Sud, F 91405 Orsay Cedex
}

\begin{abstract}
The Orientation Distribution Function (O.D.F.) calculation is usually performed using pole figures measured by X-ray or neutron diffraction. However, this kind of experimental technique does not allow to determine a total ODF since the odd terms of the series expansion are not directly accessible from pole figures. The individual orientation measurement technique can be used but it is necessary to estimate the right orientation number necessary to calculate a statistically reliable ODF. For samples at the surface, at the fifth of the thickness from the surface and at the centre of a $\mathrm{Fe} 3 \% \mathrm{Si}$ sheet, the present study shows that only 100 orientations are sufficient to find the main components of the texture but this number must be increased by a factor 10 to evaluate with a rather good accuracy the height of the peaks. In the case of a titanium alloy so called TA6V 350 orientations appear to be sufficient.
\end{abstract}

KEY WORDS EBSP, microtexture, iron and titanium alloys, even and odd ODF.

\section{INTRODUCTION}

The tensorial properties of crystals vary with the measurement direction, so they are tightly linked to the presence of preferential orientations so called crystallographic texture which acts as a weight function in the calculation of average properties of a material. The global texture of a polycrystalline material can be characterized from pole figures determined by $\mathrm{X}$ ray or by neutron diffraction which allows the Orientation Distribution Function or O.D.F. to be calculated. However this global characterization of the texture is badly adapted to the study of the mechanisms of nucleation and grain growth in recrystallization experiments; in this case, it is indeed important to know the local texture, that is, the orientation relationships between a given grain and its first neighbours. So it is more judicious to use techniques of measurements of individual orientations as T.E.M., Kossel patterns, E.C.P., S.A.C.P. or E.B.S.P. Moreover, because of the Friedel diffraction law or of the centrosymmetry of crystals, it is impossible by $\mathrm{X}$ ray or neutron diffraction to distinguish a direction $+h$ from a direction $-h$ and consequently only the even part $\tilde{F}(g)$ of the total ODF $F(g)$ is accessible.

The technique of individual orientation measurements by E.B.S.P., Dingley (1984), (1988), is perfectly adapted for solving these problems. We retained it because it is non destructive and it has good spatial resolution $(0.2-0.5 \mu \mathrm{m})$. This technique allows one to study crystalline misorientation problems at the local scale, it is indeed possible to determine the special boundary $\Sigma$ between a grain and its neighbours, which is an important parameter for grain growth, Randle et 
al. (1988), Randle et al. (1989), Rouag et al. (1989), Penelle et al. (1992). At a global scale, it is possible to calculate from a statistical point of view the respective percentages of special and general grain boundaries, Baudin et al. (1992). This technique also allows one to solve the problem of the odd part $\tilde{F}(g)$ and to calculate the total texture function $F(g)$. Finally, knowing the parameters of position, of orientation, of misorientation and of shape of the grains it will be possible to establish correlations between these different functions. However a first step consists in estimating the number of orientations required to obtain reliable results Wright et al. (1990), Baudin et al. (1992), especially concerning the O.D.F. calculation.

The aim of the present work was to characterize by E.B.S.P. the primary recrystallization texture of a $\mathrm{Fe} 3 \% \mathrm{Si}$ sheet and that of a titanium alloy TA6V and to determine for each of these two crystalline structures, cubic and hexagonal, the minimum number of grains to take into account, to calculate an O.D.F. similar to that obtained from pole figures.

A first study, Rouag et al. (1986), Vigna (1987), Rouag (1988), Rouag et al. (1989), Rouag et al. (1990), relative to the evolution of the local texture and of the speciality of grain boundaries during abnormal growth of Goss grain $\{110\}$ $\langle 001\rangle$ in $\mathrm{Fe} 3 \% \mathrm{Si}$ sheets grade $\mathrm{Hi}-\mathrm{B}$, suggests that at the primary recrystallized state, the Goss grains belong to the class of small grains and are randomly distributed through the sheet thickness and not concentrated at a fifth of the sheet thickness from the surface as it was generally assumed. In the presence of AlN and MnS inhibitors the growth of Goss grains during the secondary recrystallization annealing is due to the high mobility of the special grain boundaries $\Sigma 9$ and their subsequent expansion in subsurface is favoured by the purification of the material because of the hydrogen atmosphere used for the final annealing. However the influence of the nature of the grain boundary plane was neglected in the present approach.

In the case of the titanium alloy TA6V, only the texture of the alpha phase, which is in majority at room temperature, was determined. The studied sample was a TA6V bar forged in $(\alpha+\beta)$ field then annealed under the $\beta$ transus.

For both materials the minimum number of grains needed for statistical reliability was determined and in the case of the $\mathrm{Fe} 3 \% \mathrm{Si}$ sheet the texture gradient through the thickness was characterized, moreover the functions $\tilde{F}(g)$, $\tilde{F}(g)$ and $F(g)$ were computed.

\section{CALCULATION OF THE TOTAL O.D.F.}

The total texture function can be expressed as the sum of a function $\tilde{F}(g)$ directly determinable from pole figures and of a function $\tilde{F}(g)$ not directly determinable, with:

$$
F(g)=\tilde{F}(g)+\tilde{F}(g) \geq 0
$$

$\tilde{F}(g)$ corresponds to the series expansion on spherical harmonic bases with $l$ even 
$\left(l_{\mathrm{e}}\right)$ and $\tilde{\tilde{F}}(g)$ to the expansion with $l$ odd $\left(l_{\mathrm{o}}\right)$.

$$
\begin{aligned}
& \tilde{F}(g)=\sum_{l_{\mathrm{c}}} \sum_{m} \sum_{n} f_{l m n} T_{m n}^{l}(g) \\
& \tilde{F}(g)=\sum_{l_{\mathrm{o}}} \sum_{m} \sum_{n} f_{l m n} T_{m n}^{l}(g)
\end{aligned}
$$

where the $T_{m n}^{l}(g)$ are the generalized spherical harmonics.

From $N$ orientations $g_{i}$, the coefficients $f_{l m n}$ of these equations can be calculated using the relation proposed by Bunge (1982):

$$
f_{l m n}=\frac{1}{N} \sum_{i=1}^{N} K T_{m n}^{l^{*}}(g)
$$

The asterisk means the conjugate part with

$$
K=\frac{\exp \left(-l^{2} \Phi_{0}^{2} / 4\right)-\exp \left[-(l+1)^{2} \Phi_{0}^{2} / 4\right]}{1-\exp \left(-\Phi_{0}^{2} / 4\right)}
$$

The expression of the parameter $K$ proposed by Truszkowski et al. (1973), allows each orientation to the modelled by a gaussian characterized by the parameter $\phi_{0}$ linked to the half height width $b$ of this gaussian by the relation:

$$
\Phi_{0}=b / 2 \sqrt{\ln 2}
$$

Then it is necessary to estimate as well as possible the value of $b$ or of $\phi_{0}$; Wagner (1983) proposed to approximate $\phi_{0}$ using an expression which takes into account the number of measured orientations $N$ and the sharpness of the texture through a parameter $p$

$$
\begin{gathered}
\Phi_{0}=(\pi p / 2 N)^{1 / 3} \text { for cubic structure } \\
\Phi_{0}=(\pi p / N)^{1 / 3} \text { for hexagonal structure }
\end{gathered}
$$

If the texture is isotropic, $p$ is equal to 1 whereas $p$ tends to 0 for a sharp texture. In general, in the case of soft texture a value equal to 0.5 is generally retained, Wagner (1983), Wright et al. (1990).

So it can be seen that the expansion order $L$, the number of grains $N$ and $\phi_{o}$ which depends on the sharpness of the texture $p$ and of $N$, are parameters which have to be optimized; in the present study $p$ was equal to 0.5 .

This method for calculating $\tilde{F}(g)$ and $\tilde{F}(g)$ is based on the use of the harmonic method which leads to truncation errors since the expansion order is limited to a finite value $L$. To avoid this problem the Matthies method provides an exact solution, for that purpose, Matthies et al. (1987) introduced the Gauss shaped standard function.:

$$
F(S, \omega)=N(S) e^{S \cos \omega} \geq 0
$$

with $\omega=\omega\left(g_{0}, g\right)$ and $0 \leq S<\infty$

$F(S, \omega)$ represents the total texture function (or $F(g))$ calculated for a given orientation $\left(g_{0}\right) . N(S)$ is a constant of normalisation calculated using Bessel functions, Matthies et al. (1987). The $S$ parameter allows us to characterize the $g_{0}$ orientation shape. For $S=0$, Eq. (8) describes a random distribution and if $S$ tends to infinity, the orientation possesses the properties of a Dirac function. $S$ is 


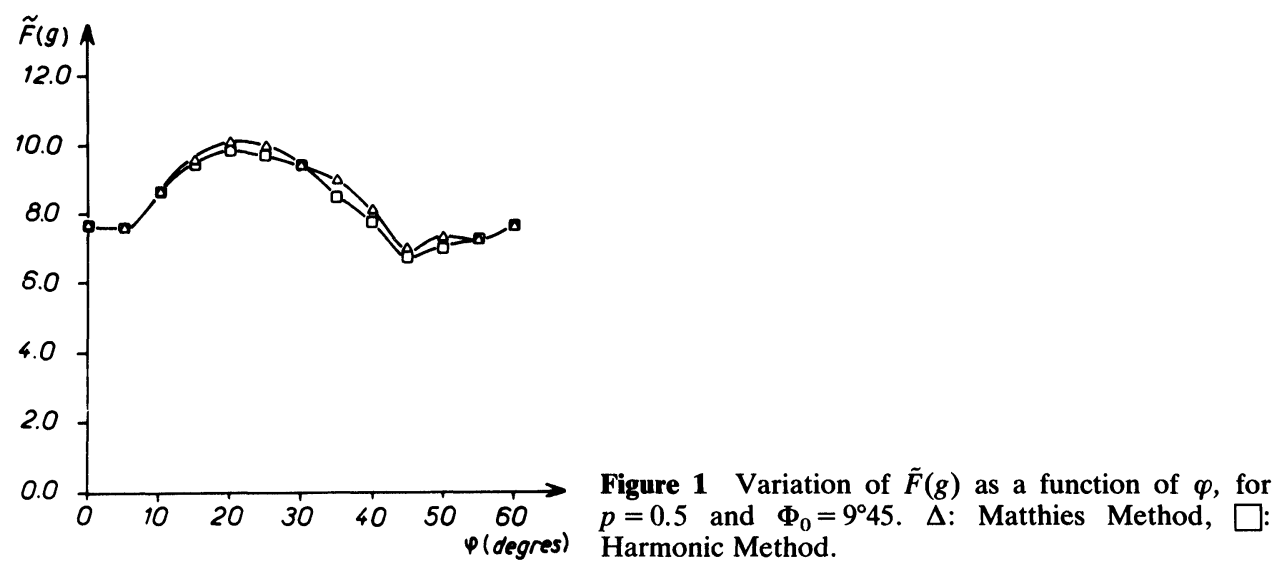

linked to the $b$ parameter by the relation:

$$
S=\ln 2 /\left[2 \sin ^{2}(b / 4)\right]
$$

with $b \leq 2 \pi$.

If $b \ll \pi(S \gg 1)$, Eq. (8) becomes equal to that proposed by Bunge (1982) to model a classic Gauss distribution:

$$
F\left(\Phi_{0}, \omega\right)=N\left(\Phi_{0}\right) e^{-\left(\omega / \Phi_{0}\right)^{2}}
$$

This approach also allows, $\tilde{F}(g)$ and $\tilde{F}(g)$ to be calculated using $F(g)$ and a ghost function $F_{G}$ expressed with Bessel functions:

$$
\tilde{F}(g)=F(g) / 2+F_{G}(g)
$$

Both methods have been used to calculate $\tilde{F}(g)$ and $F(g)$. An example is given for the titanium alloy sample TA6V with 350 grains which is a sufficient number as will be shown later; the aim of such a calculation was to verify that $L=16$ is an adequate expansion order. So the Figures 1 and 2 show respectively the evolution

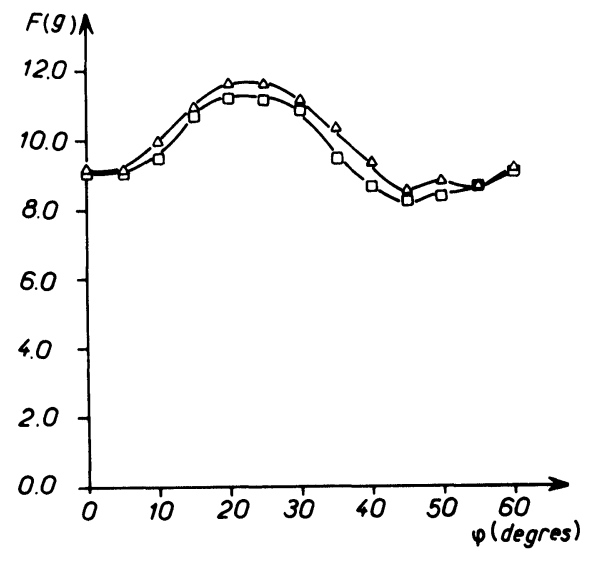

Figure 2 Variation of $F(g)$ as a function of $\varphi$, for $p=0.5$ and $\Phi_{0}=9^{\circ} 45 . \Delta$ : Matthies Method, Harmonic method $(L=16)$. 
of $\tilde{F}(g)$ and $F(g)$ as a function of the Euler angle $\varphi$ in the Roe notation, Roe (1965), (1966). As can be seen, the two methods lead to very close results so that it is reasonable to think that $L=16$ is sufficient for the present texture, which is in agreement with the results of Dervin (1977), and Naka et al. (1977). Complementary calculations at $L=34$ give results similar to those obtained with $L=16$, as an example $\tilde{F}(g)=-1.17$ for $L=16$ and -1.30 for $L=34$; difference between both values is due to truncation errors, (with Matthies method $\tilde{F}(g)=-1.30$ ). Let us note that $L=22$, produces satisfactory results for the Fe $3 \%$ Si sample.

\section{RESULTS}

\section{III.1. Primary Recrystallization Texture in $\mathrm{Fe} \mathrm{3 \%} \mathrm{Si}$}

The aim of this section was to compare global and local textures measured by $\mathrm{X}$ ray diffraction (pole figures) and by EBSP on three samples, at the surfaces, at a
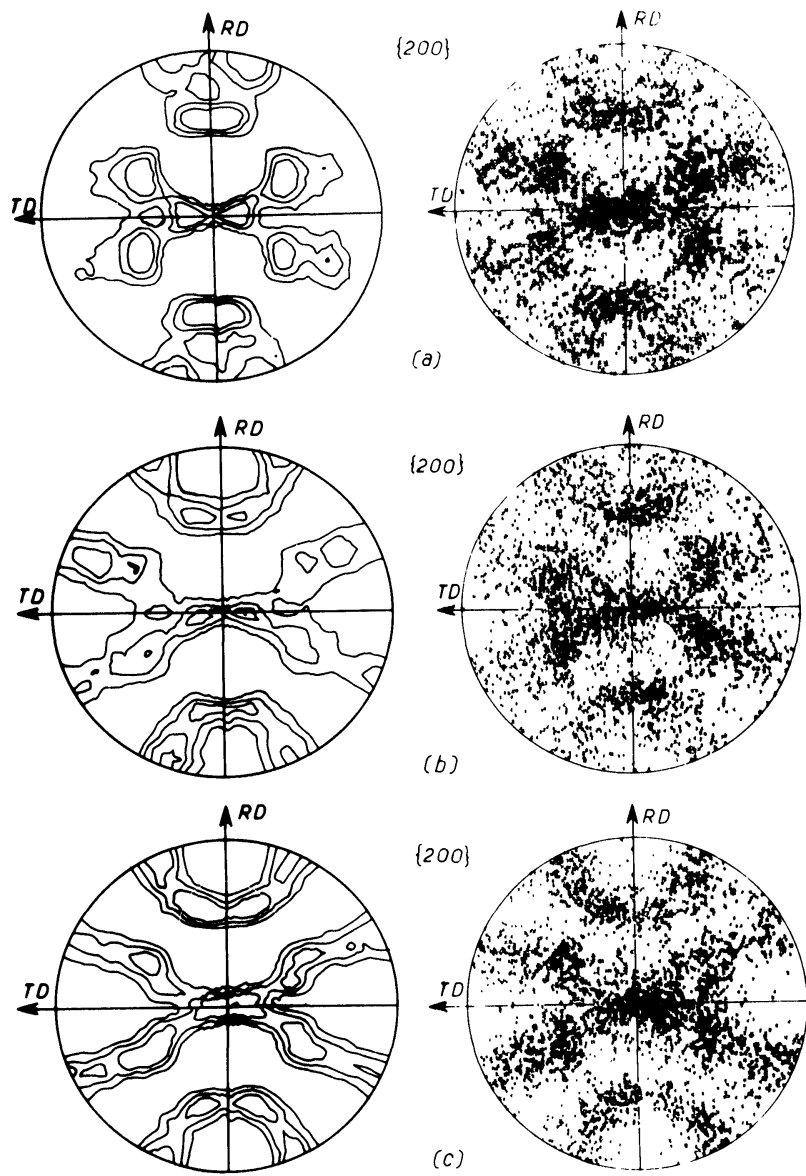

Figure $3\{200\}$ pole figures. Comparison between $\mathrm{X}$ ray diffraction and EBSP. a-surface, $\mathrm{b}-\mathrm{fifth}$, c-center. 
fifth of the sheet thickness from the surface and at the center, Rouag (1988), Penelle et al. (1992), Baudin et al. (1992).

The EBSP technique allows one to identify the Goss grains through the sheet thickness, to calculate their volume fraction and also to estimate the gradient of each component.

For each sample (surface, fifth, center), 1000 orientations were measured and it is important to underline that, among the 3000 orientations, only one Goss grain within about $1.5^{\circ}$ was found and that at the center of the sheet. Figure 3 shows the $\{200\}$ pole figures determined by X ray diffraction and by EBSP; qualitatively a good agreement is observed between these different pole figures. This agreement indicates that qualitatively good statistics are obtained with 1000 grains.

The Figures $4 \mathrm{a}, \mathrm{b}, \mathrm{c}$ allow us to compare the total functions $F(g)$ for the samples at the surface, at the fifth and at the center for an expansion order $L=22$ (the same as the one used for pole figures); these sections at $\varphi=45^{\circ}$ show that the sharpness of $F(g)$ for the component $\{111\}\langle 112\rangle(\psi=0$ and $60, \theta=55$, $\left.\varphi=45^{\circ}\right)$ decreases from the surface to the center whereas $F(g)$ increases from the surface to the center for the orientation $\{100\}\langle 012\rangle,\left(\psi=20^{\circ}, \theta=0, \varphi=45^{\circ}\right)$. This evolution is similar to that observed for the even part $\tilde{F}(g)$ calculated from classical pole figures and for the same expansion order $L=22$. Let us note that the odd part $\tilde{F}(g)$ corresponds to about $20 \%$ of the total ODF $F(g)$. These results are summarized in Figure 5 which represents the variation of $\tilde{F}(g)$ and $F(g)$ from the surface to the center of the sheet.

In a first approach, results obtained by X-ray diffraction and by EBSP seem qualitatively in good agreement, however from a quantitative point of view it

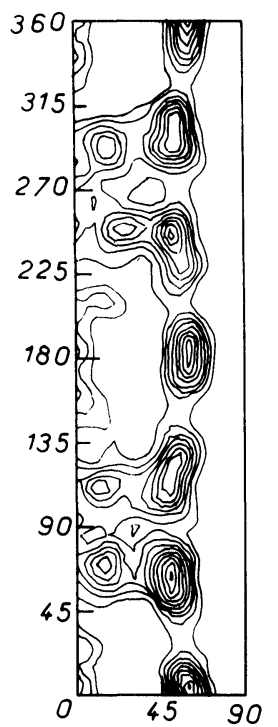

a)

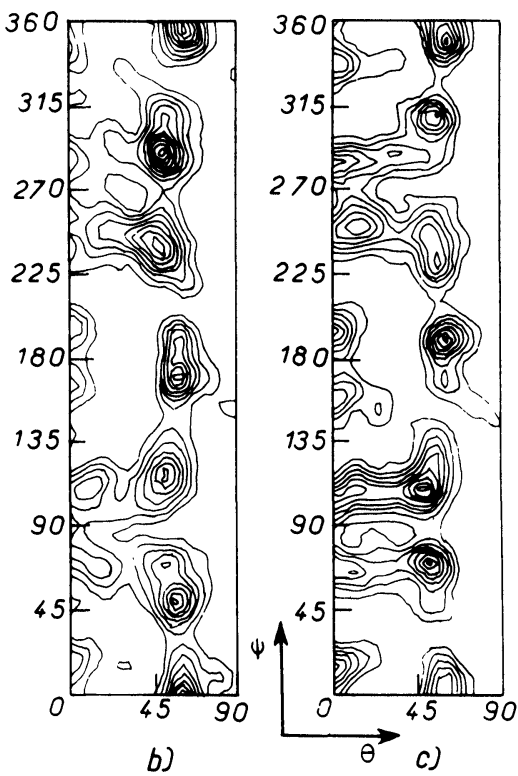

Figure $4 \varphi=45^{\circ}$ section plots of $F(g)$. a-surface, b-fifth, c-center. Levels: $1=\min , 10=\max$, step $=1$. 


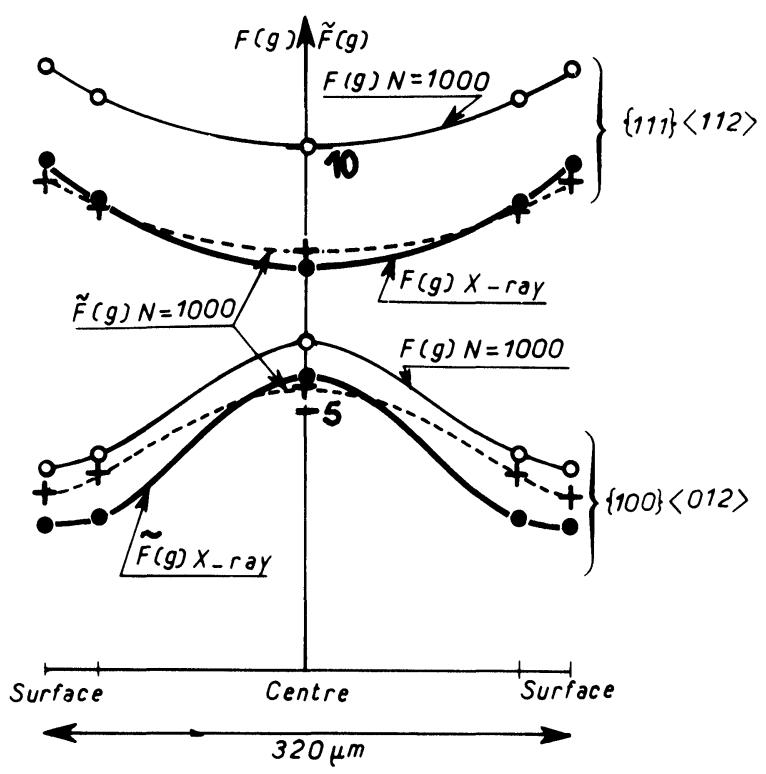

Figure 5 Texture gradient through the sheet thickness.

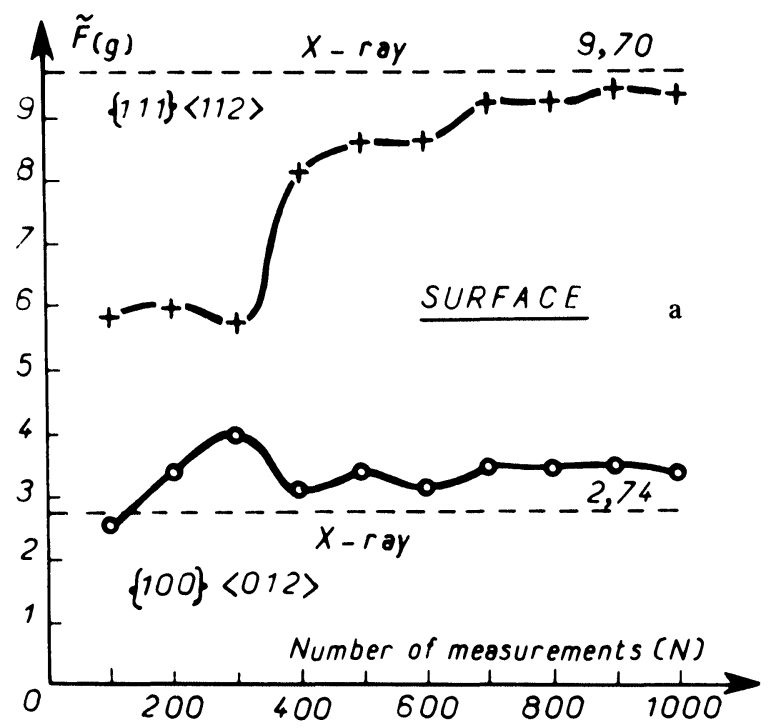

Figure 6 Variation of $\tilde{F}(g)$ as a function of the number of measurements. a-surface, b-fifth, c-center. 

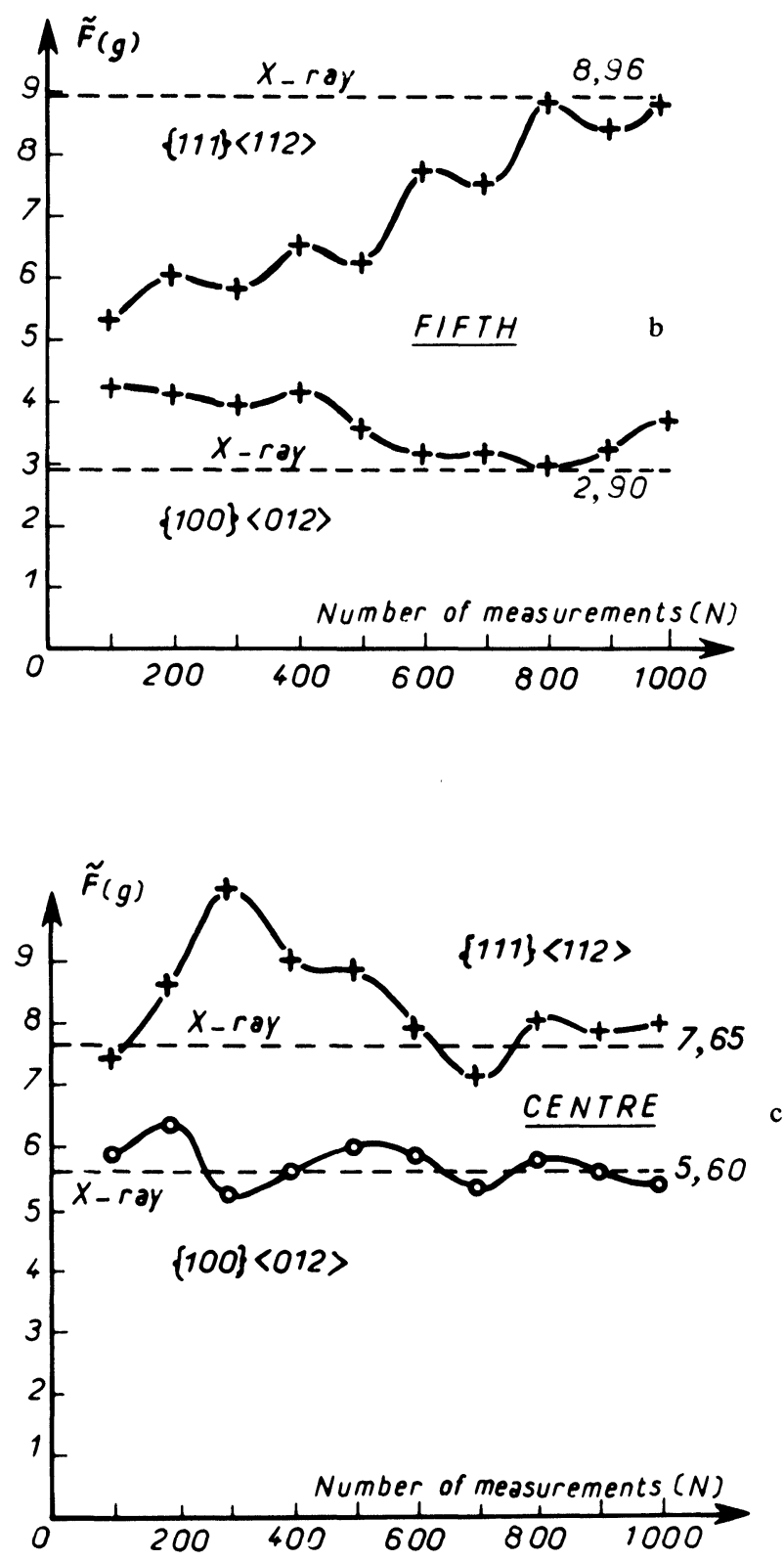

Figure 6 Variation of $\tilde{F}(g)$ as a function of the number of measurements. a - surface, b-fifth, c-center. 


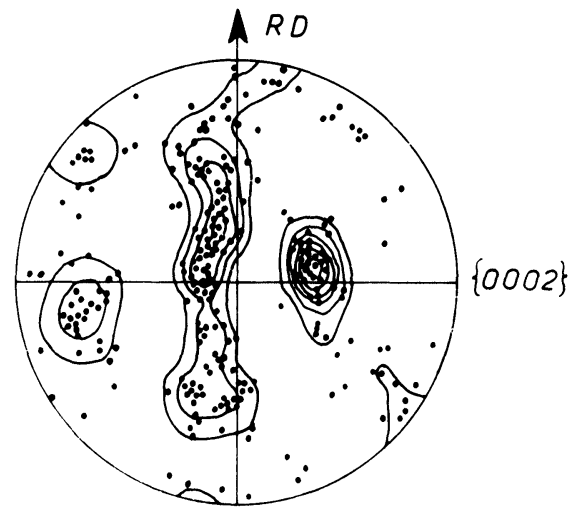

Figure $7\{0002\}$ pole figure of the TA6V sample. • individual orientations measured by EBSP, - pole density levels calculated from EBSP measurements.

appears very important to study the influence of some parameters such as the number of orientations taken into account to calculate an ODF.

In order to estimate the optimum number of grains $N$ to consider, we plotted the evolution of the maximums of $\tilde{F}(g)$ as a function of $N$. Figure 6 shows the curves $\tilde{F}(g)-N$ for the three samples, which point out instabilities up to about 700-750 measurements but after that they tend towards a constant value of $\tilde{F}(g)$ which is similar to that calculated from pole figures. However, it seems that this stability is not perfectly reached, (see Figure 6b), meaning that 1000 orientations may not be sufficient. From these figures one could think that a correct evaluation

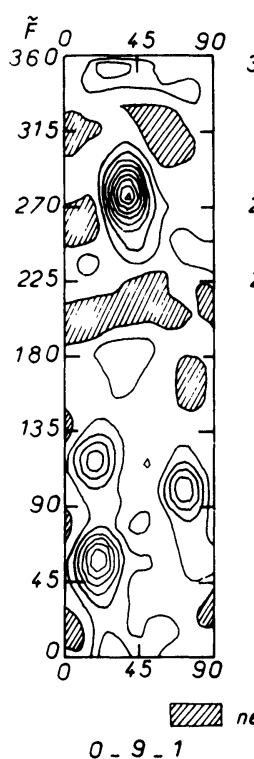

$0-9-1$

a

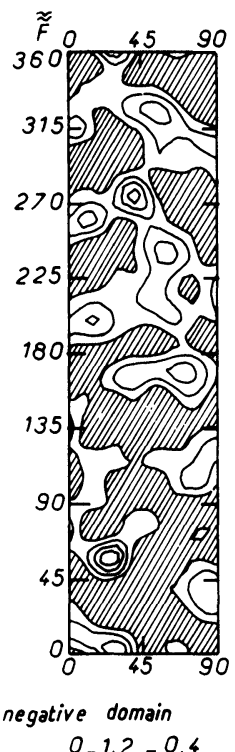

$b$

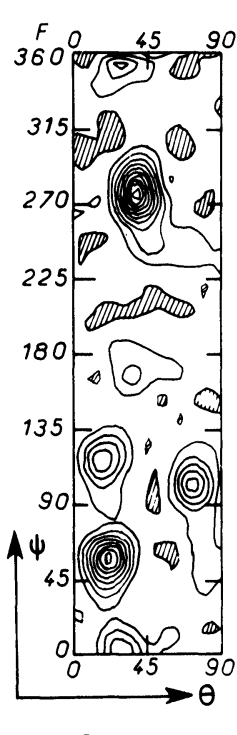

$c$

Figure $8 \varphi=30^{\circ}$ section plots of the TA6V sample. $\mathrm{a}-\tilde{F}(g), \mathrm{b}-\tilde{\tilde{F}}(g), \mathrm{c}-F(g)$. Levels: $0=\min$, $9=\max$, step $=1$. 
of the $\{100\}\langle 012\rangle$ component is obtained with a smaller number of measurements than in the case of the $\{111\}\langle 112\rangle$ component. The examination of the curves seems however to indicate that convergency is reached for a great number of grains, the observed fluctuations are due to statistical problems.

\section{III.1-2. $T A \sigma V$}

350 oreintations were measured, Figure 7 shows a $\{0002\}$ pole figure in which are reported the $350 c$ axes and the isodensity levels calculated from these 350 individual orientations. This figure points out the consistency of the calculations of the pole density.

From these measurements, the $\tilde{F}(g), \tilde{F}(g)$ and $F(g)$ ODFs have been computed and the sections of each at $\varphi=30$ are given in Figure 8 . The texture is complex but it can be roughly interpreted with the three components $(\overline{2} 114)$

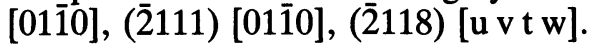
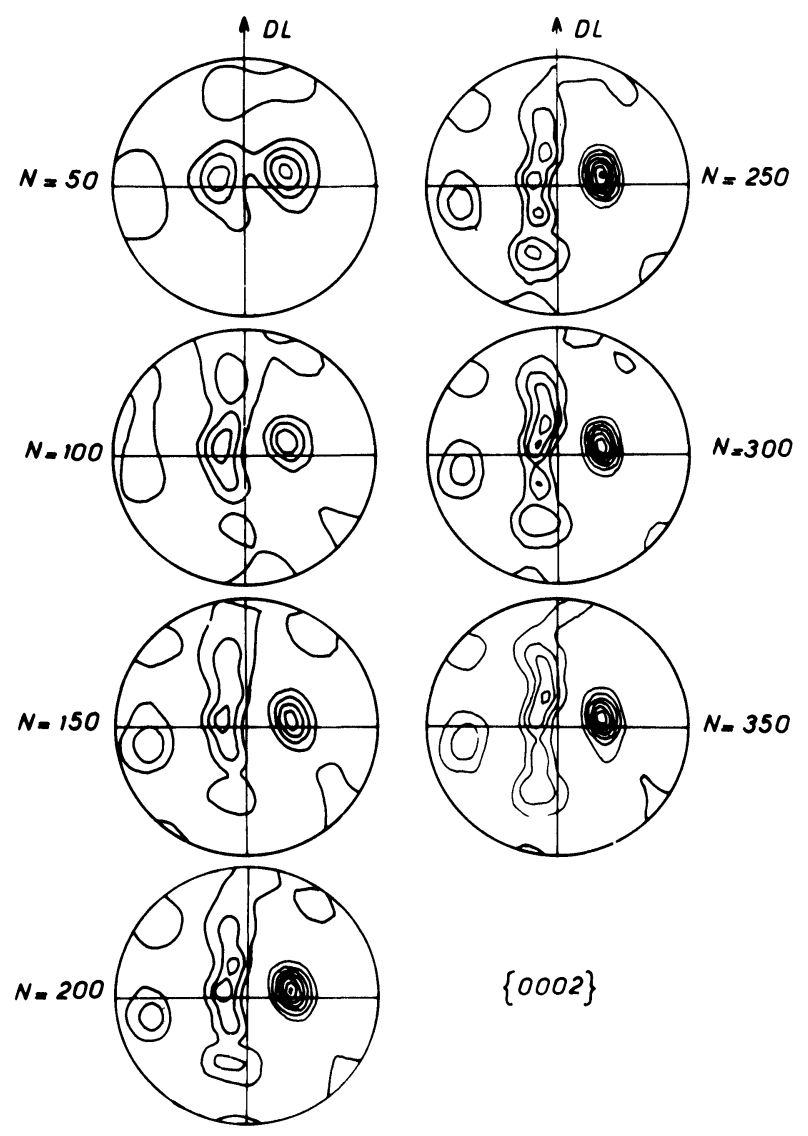

\section{$\{0002\}$}

$$
-1-7-1-
$$

Figure 9 Evolution of the $\{0002\}$ pole figures as a function of $N$ levels: $1=\min , 7=\max , \operatorname{step}=1$. 


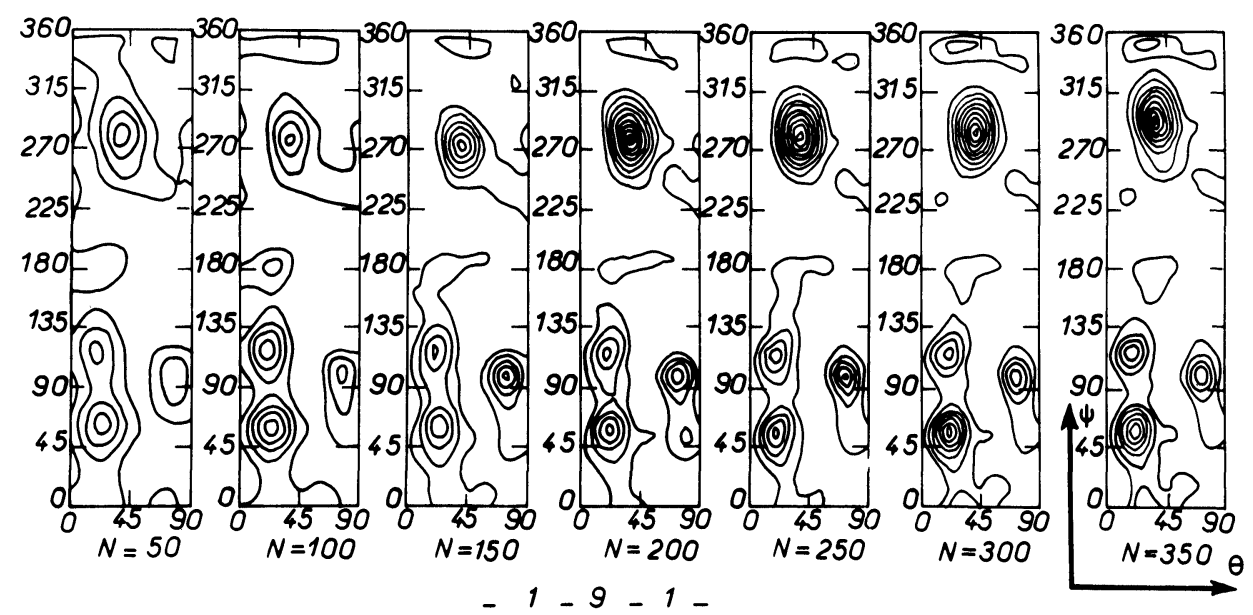

Figure 10 Evolution of the section plots of $\tilde{F}(g)$ versus $N$. Levels: $1=\min , 7=\max$, step $=1$.

In order to determine the influence of the number of orientations, we have calculated the $\{0002\}$ pole figures using $50,100,150 \ldots 350$ grains, Figure 9 . As can be seen the pole figure calculated with 50 grains is very different from that calculated with 350 grains, this fact is confirmed by Figure 10 which represents the evolution of the $\tilde{F}(g)$ sections at $\varphi=30^{\circ}$ as a function of $N$. From this figure, it appears that at least 200 grains are necessary to obtain consistent results. To check this point, the maximum of $\tilde{F}(g)$ and $F(g)$ are plotted as functions of $N$ in Figure 11 and similarly the maximum pole density $q$ in the $\{0002\}$ pole figure is plotted in Figure 12. These two figures confirm that convergency is reached for about 200-300 grains.

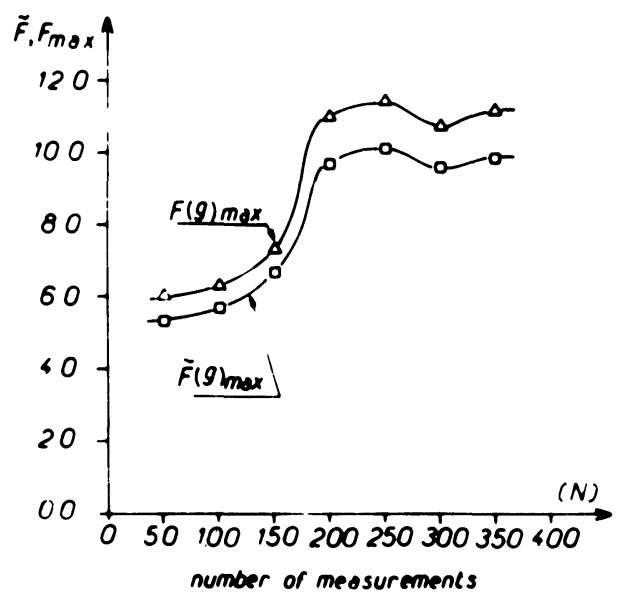

Figure 11 Variations of $\tilde{F}(g) \max -N$ and of $F(g) \max -N$. 


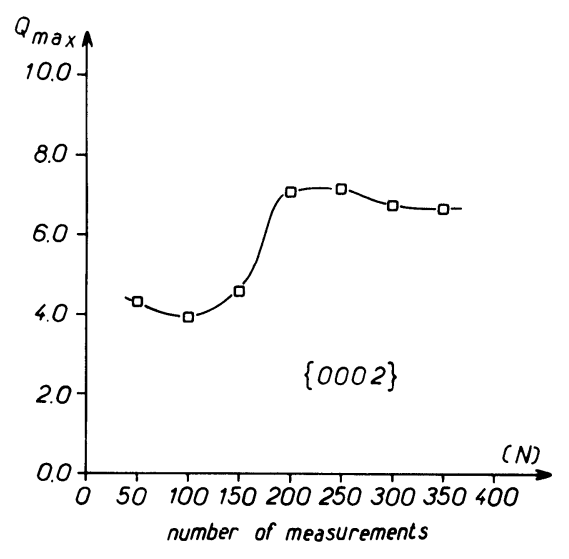

Figure 12 Variation of the maximum of the pole density $q$ as a function of $N$.

\section{CONCLUSION}

Although the technique of individual orientation measurement has not to be used as routinely one as X-ray or neutron diffraction, it allows one to verify numerical methods used to calculate $F(g), \tilde{F}(g)$ and $\tilde{F}(g)$. The present study focused on the evaluation of the minimum number of orientation measurements needed to calculate a consistent ODF. It appears that about 1000 grains are necessary in the case of the $\mathrm{Fe}$ 3\% Si sheet whereas only 300-350 grains are sufficient for the TA6V sample, however for this last material it would be worth confirming this result using a greater number of orientations. Nevertheless, it is difficult to definitely conclude, because of the difference of crystalline symmetry, the difference in texture and the difference in grain size of the two materials.

\section{References}

Baudin, T., Paillard, P. and Penelle, R. (1992). J. of Appl. Cryst., 25, 400-408.

Bunge, H. J. (1982). Texture analysis in Materials Science, Butterworth Pub. London.

Dervin, P., Mardon, J. P., Pernot, J., Penelle R. and Lacombe, P. (1977). J. Less Common Met., 55, 25.

Dingley, D. J. (1984). Scanning Electron microscopy, 2, 569.

Dingley, D. J. (1988). Proc. ICOTOM8, Santa Fe USA., September 20-25, Ed. J. S. Kallend, G. Gottstein, The Metallurgical Society, 189.

Matthies, S., Vinel, G. W. and Helming, K. (1987). Standard distribution in Texture Analysis, Akademic-Verlag Berlin.

Naka, S., Mardon, J. P., Dervin, P., Pernot, M., Penelle, R. and Lacombe, P., (1977). J. Less Common Met., 55, 277.

Penelle, R., Baudin, T., Paillard, P. and Mora, L. (1992). Proc. ICOTOM9, Avignon, France, 17-21 September 1990, Textures and Microstructures, 1991, vols 14-18, pp. 597-610.

Randle, V. and Ralph, B. (1988). Textures and Microstructures, 8-9, 531.

Randle, V. and Brown, A. (1989). Phil. Mag., 59, 5, 1075.

Roe, R. J. (1965). J. Appl. Phys., 36, 2024.

Roe, R. J. (1966). J. Appl. Phys., 37, 2069.

Rouag, N., Vigna, G. and Penelle, R. (1986). Proc. 7th Int. Symp. on Metallurgy and Material Science, Riso, Denmark, 521.

Rouag, N. (1988). "Influence de la texture cristallographique et de la spécialité des joints de grains sur l'anisotropie de migration des joints entourant un grain d'orientation $\{110\}\langle 001\rangle$ au cours des premiers stades de la recristallisation secondaire dans des tôles $\mathrm{Fe} 3 \% \mathrm{Si}$ en présence de précipités AlN et MnS", Thèse de Doctorat d'Etat, Orsay. 
Rouag, N. and Penelle, R. (1989). Textures and Microstructures, 11, 203.

Rouag, N., Vigna, G., Penelle. R. (1990). Acta Metal., Mater., 38, 6, 1101.

Truszkowski, W., Pospiech, J., Jura, J. and Major, B. (1973). 3ème colloque européen sur les textures de déformation et de recristallisation des métaux et leurs applications industrielles, Pont-á-Mousson, France, 235.

Vigna, G., (1987), “Application des techniques de canalisation d'électons et de Kossel à l'étude de la texture locale et des contraintes internes de tôles Fe 3\% Si", Thèse de Doctorat en Sciences, Orsay.

Wagner, F. (1983). "Contribution à l'étude des modifications de texture par déformation et transformation dans les matériaux de basse symétrie". Thèse d'Etat, Metz.

Wright, S. I. and Adams, B. (1990). Textures and Microstructures, 12, 65. 